\title{
Health Monitoring Using Cloud based Internet of Things
}

\author{
K.Laxmareddy, K.Archana, Y.Gayathri, C.Karthik
}

\begin{abstract}
The proposed structures for far flung properly being looking offer three diploma engineering. A far flung body place network (WBAN), incorporates of wearable sensor because the facts acquision unit, correspondence and organizing, and the management layer. The framework is supposed for lengthy haul stockpiling of patient's biomedical data too helping health specialists with symptomatic data.

The wearable sensors that degree the physiological parameters, as an example, circulatory strain, inner warmth degree are related to the system thru concentrators (PDA). The sensor statistics is transmitted to a passage server thru Bluetooth affiliation. The passage server transforms the records right into a notion and the executives file and stores it on a much flung server for later recovery by using clinicians through the internet. Cloud getting ready has 3 unmistakable elements: stockpiling, examination, and representation. The information of the sufferers is located away in cloud for long haul. Investigation makes use of the sensor statistics alongside e-health information and assists with finding and visualizations of infections. Notion makes the records and dissect open to the doctors in a proper away absorbable enterprise.

No matter the reality that there are numerous benefits of allotted computing. Anyhow, it has safety strings of essential non-public information. Customers of cloud innovation can not rely upon the cloud management sup-forceps for the safety of the important thriller information. On this way, a third-birthday party Authenticator is needed which confirms the cloud records from the side of clients or holders of the data. Safety of sensitive information is of faulty nature due to the nearness of various substances. Cloud service carriers alongside facts users are further on top of things for putting the safety of the critical thriller data in threat.
\end{abstract}

Keywords-wearable sensors, IoT, illustration, studies, cloud, protection..

\section{INTRODUCTION}

During the most current decade, net of things (IoT) has pulled in focused attention because of a massive scope of utilizations in modern-day, biomedical notion, farming, sensible city areas, herbal checking and unique fields (Fig. 1) [1]. IoT is the internetworking of bodily gadgets carried out in our every day revel in that utilization extensive correspondences fashions to present new administrations to give up customers [2]. From an inexpensive outlook, the

Revised Manuscript Received on September 14, 2019.

K.Laxmareddy Professor, Department of ECE, Siddhartha Institute of Technology \& Sciences. Hyderabad, Telangana, India.

K.Archana, Assist. Prof, Department of ECE, Siddhartha Institute of Technology \& Sciences, Narapally, Ghatkesar, Hyderabad, Telangana, India.

Y.Gayathri Assist. Prof, Department of ECE, Siddhartha Institute of Technology \& Sciences, Narapally, Ghatkesar, Hyderabad, Telangana, India.

C.Karthik Associate professor Department of ECE, Affiliated to JNTU colleges, Hyderabad, Telangana, India. (E-mail: Karthik.kamalesh@gmail.com) additives of IoT abridged into a sincere circumstance beneath

IoT $=$ Human + bodily items (sensors, controllers, gadgets, stockpiles) + net

It is imagined that by the usage of 2020 the destiny internet will contain many billions of eager articles/gadgets [3]. IoT innovation gives higher administrations to cease customers by means of the use of ongoing information approach-ing, interchanges and perception. IoT may be stretched out to almosteverything from refrigerator to clothes washing machine, wristwatches to cell telephones, domestic safety to alert tool,and plenty of others [4]. As an instance, savvy coolers can reveal to us the finish of the legitimacy of nourishment using standardized identifications or which topics to buy in the course of our purchasing within the marketplace. Alternatively, envision that we can control our home from wherever. By utilizing advanced cell telephones or drugs with clearly primary contact we can set a super temperature or flip lights on or off before returning domestic. These are times of most effective multiple utilizations out of thousands being as of now constructed up each day within the place of IoT.

The IoT is a megatrend in cutting facet advances that could have an effect on the whole employer range and can be concept of as the interconnection of enormously recognizable clever items and gadgets inside the present net framework with extended advantages. Blessings commonly include the propelled availability of these gadgets, frameworks, and administrations that is going beyond system-to-gadget (M2M) conditions [5]. In this manner, offering computerization is possible in almost each region. The IoT gives becoming answers for a wide scope of utilizations, as an instance, savvy urban areas, web site visitors blockage, squander the board, auxiliary health, protection, disaster administrations, coordinations, retails, cutting-edge manipulate, and medicinal services. The intrigued peruser is alluded to [5] [6] for a extra profound comprehension of the IoT.

Therapeutic interest and medicinal services speak to one of the maximum captivating software program zones for the IoT [7]. The IoT can possibly provide ascent to severa therapeutic packages, for example, far off well-being gazing, exercise session regimes, ceaseless illnesses, and older consideration. Consistence with remedy and drug at home and with the resource of social insurance vendors is a few different large capability utility. Alongside these strains, wonderful restorative gadgets, sensors, and analytic and imaging devices may be seen as keen devices or articles 
comprising a center piece of the IoT. IoT-based human services administrations are required to decrease prices, increment the personal pleasure, and enhance the client's expertise. From the viewpoint of human services suppliers, the IoT can probable decrease machine excursion via a ways flung arrangement

There may be a need of a propelled model for protection, which considers the security issues from a comprehensive for each spective concerning the propelled customers and their between correspondence with this innovation. Web is prima-ry of IoT henceforth there can be safety proviso. Among correspondence excellent fashions are created depending on detecting programming for IoT programs, advancing an intercommunication stack to build up the crucial effectiveness and unwavering notable. Verifying intercommunication is an important hassle for each one of the standards which are developing relying on detecting programming for IoT packages. Security escape clauses may be decided in discernment layer, bodily layer, installation layer and alertness layer. As of now IP-based totally intercommunication worldview of internet structure assumes a critical challenge in building up the pervasive interconnectivity of customers and devices via IoT

\section{SYSTEM ARCHITECTURE \& RESULTS}

Figure 1 illustrates the system architecture for a remote health monitoring system

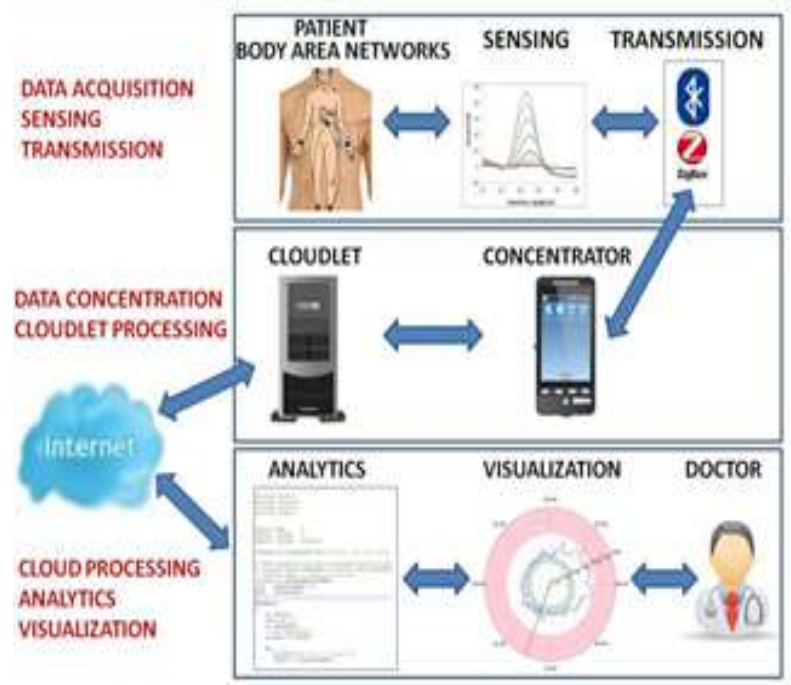

Fig. 1. Remote patient monitoring system based on an IoT-Cloud architecture

\section{A. Data Acquisition:}

Records acquisition was finished with several wearable sensors that measure physiological biomarkers, such as ECG, frame position and temperature. Sensors related to the community despite the fact that the intermediate data aggregator or concentrator, which is usually smart phones are located around the patient

(i) ECG SENSOR

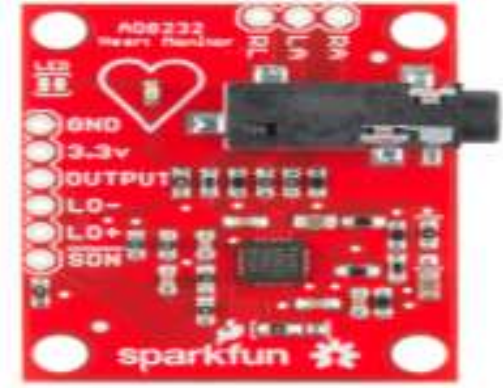

Fig 2: ECG Sensor

Figure 2 indicates that the EKG sensor. This sensor is applied to gauge the board feel the heart's electrical development. This electric development may be portrayed as an ECG or electrocardiogram. AD8232 is a karmic block forming composed sign for ECG and different biopotential estimation utility. Figure four indicates that the ECG waveform. ECG can to an vast degree difficult, AD8232 Lead Single Heart Rate Monitor is going about as amp activity to assist get an unmistakable signal of compelling PR and QT intervening time. ECG sensor associated with the ADC. ECG pulse sensor detects and send it to the microcontroller

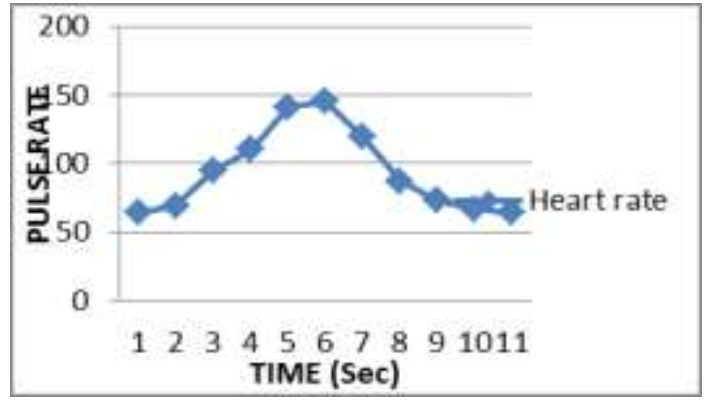

Fig 3 Waveform Generation of Heart Rate

Pulse is one of the most much of the time estimated parameter of the human body and assumes a significant job in deciding a person's wellbeing. Pulse is the quantity of pulses per unit of time, normally communicated as beats every moment (bpm). pulse can fluctuate as indicated by the necessities of the body to ingest oxygen and radiate carbon dioxide changes, for example, during activity or rest. Figure 5 shows that the investigation and exchange in the age of waves from the patient's pulse

\section{(Ii) Principles of Body Position}

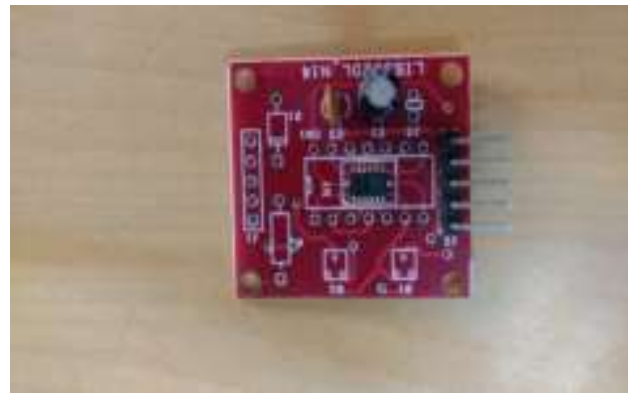

Figure 3: Body Position Sensor 
Figure (3) shows that the accelerometer is a gadget that estimates valid dashing up. Quickening proper, into increasing the velocity (or pace the tempo of development) of the body within the the rest of the fast line itself, isn't equivalent to set quickening, becoming quicken the settling framework encourages. MEMS Accelerometer sensor associated with the $\mathrm{I} 2 \mathrm{C}$ and is utilized to decide the subtleties of the affected person's frame position. Factor place calculation applies to the usual of body position. This calculation is applied to decide the scenario of the affected person

(iii) Body Temperature sensor

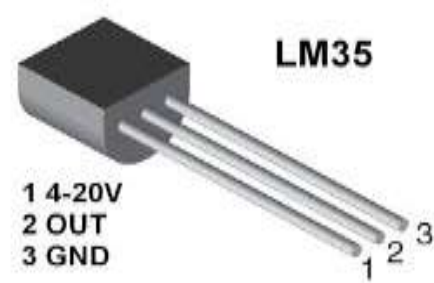

Fig 4 Temperature Sensor

Figure (four) shows that the LM35 is a joined circuit sensor that may be used to quantify temperature with an electrical yield regarding the temperature (in ${ }^{\circ} \mathrm{C}$ ). It may gauge temperature more decisively than a the usage of a thermistor. The sensor device is consistent and no longer divulge to oxidation. Temperature sensor related to ADC stick. This sensor sense the affected person's internal warmness stage.

\section{B. Records TRANSMISSION}

The facts Transmission parts of the framework are chargeable for passing on chronicles of the affected individual from the affected man or woman's domestic (or any a ways off area) to the server farm of the Healthcare company (HCO) with assured protection and safety, in an awesome global in close to ongoing. Typically, the tactile obtaining degree is provided with a short range radio such

As Zigbee or low-manipulate Bluetooth, which it uses to move sensor facts to the concentrator. Totaled data is furthermore surpassed-off to a $\mathrm{HCO}$ for long haul stockpiling utilising internet connec-tivity on the concentrator, often via a cellular mobile phone's WiFi or mobile

Facts affiliation. Sensors in the records procurement element shape a web of things (IoT)- primarily based totally layout as each person sensor's records may be gotten to via the net by means of using the concentrator [8], [9].

\section{Information attention}

Often a potential/handling machine in location of a portable consumer, once in a while alluded to as a cloudlet, is applied to amplify its stockpiling/getting ready capability at a few factor point the neighborhood versatile assets do now not fulfill the software program's stipulations [10]. The cloudlet can be a close-by making ready unit, (as an example, a laptop) this is legitimately to be had with the aid of the concentrator via WiFi set up. However giving quick stockpiling preceding correspondence of records to the cloud, the cloudlet can likewise be applied for on foot time easy undertakings at the affected person's collected records. Further, the cloudlet can be implemented to transmit the collected records to the cloud if there should be an prevalence of confinements at the mobile telephone, for instance, transitory absence of availability or energy.

\section{CLOUD PROCESSING}

Cloud Processing has three specific segments: stockpiling, investigation, and belief. The framework is meant for lengthy haul stockpiling of patient's biomedical records just as sisting health experts with symptomatic statistics. Cloud primarily based medicinal records stockpiling and the forthright troubles have been significantly tended to inside the writing [11], [12]. Analyticsthat utilize the sensor information along e-health statistics thatare reworking into pervasive can help with determinations and guesses for severa fitness situations and ailments. Moreover, Visualization is a key prerequisite for this form of framework becauseit is unrealistic to request that medical doctors pore over the voluminous data or investigations from wearable sensors. Representation strategies that make the facts and examinations to be had to them in a without delay suitable for eating association are simple if the wearable sensors are to have an effect on scientific exercise.

\section{DANGERS TO THE SAFETY INSIDE THE NET OF FACTORS}

Protection dangers to the IoT are fundamentally of three kinds on premise of the assault aim.

1. Assaults in competition to an IoT machine,

2. Assaults in competition to the specialised (device server) channel

Three. Assaults toward the server.

\section{A. Assaults in opposition to IoT gadgets}

For an assailant, it's miles workable that an IoT device with pre-gadgets is a clean and full-size prey for a few motives. For instance An associated safety framework added at domestic each time traded off, may want to give restricted safety facts about the repute of a given vicinity.

\section{B. Assaults towards Communications}

Checking and improving the messages while they may be being imparted may be a way to jeopardize the safety of IoT. There may be a outstanding extent of statistics that is crossed thru the IoT scenario which makes it increasingly more risky because the messages can be managed while in travel.

\section{Attacks toward the grasp of gadgets}

The ace framework problems and oversees information examination of IoT gadgets certainly as offers with them. The bosses consisting of makers, CSPs, and IoT association suppliers - whilst assaulted may be incurred with excessive damage. These are alloted with an entire lot of personal facts, a number of which can be profoundly touchy. 


\section{Attacks on notion Layer}

Maximum decreased layer in IoT and it's miles wellspring of information. Safety problems can be discovered in physical detecting gadgets and assortment of facts. Protection attacks on WSN, which locate and manage the earth can be Secrecy and confirmation, management respectability and gadget accessibility. Security attack on RFID and observation layer are everyday, similar to information spillage, replay attacks, statistics following, converting, cloning attacks and trap portal hub, physical capture, uncalled for attacks, blockage attack, DoS assaults hub replication assault and for-ward assault, personally.

\section{E. Assaults on physical Layer}

Willpower and age of bearer recurrence is in line withframed thru this residue. Sticking and Node Tampering are being utilized to assault bodily layer.

\section{F. Attacks on community Layer}

Unlawful get admission to, listening in of labeled statistics, contamination and so forth are completed in set up layer. IoT is related to vast variety of gadgets, so numerous configurations are applied to gather statistics, and information is colossal and gathered from multi-property. Device blockage should have come approximately due to statistics pass, which brings approximately DoS assaults.

\section{G. Assaults in utility Layer}

Listening in and altering are massive protection issues in software program layer. Visitors the board is accomplished on this layer.

Table 1: Cloud computing threats

\begin{tabular}{|l|l|}
\hline Threat & Description \\
\hline Data breaches & $\begin{array}{l}\text { Usage of protected data and violation } \\
\text { of security }\end{array}$ \\
\hline Data loss & Loss of data due to improper handling \\
\hline Account or service traffic hijacking & $\begin{array}{l}\text { Attacks on the system to hijack the } \\
\text { data }\end{array}$ \\
\hline Insecure API & $\begin{array}{l}\text { Attacks on code signing keys used by } \\
\text { web and cloud for identification }\end{array}$ \\
\hline Denial of service & Denial of access to user \\
\hline Malicious insider activities & $\begin{array}{l}\text { Any insider can use the system for } \\
\text { malicious }\end{array}$ \\
\hline Abuse of cloud service & $\begin{array}{l}\text { Using cloud services for illegal } \\
\text { activities }\end{array}$ \\
\hline Insufficient diligence & $\begin{array}{l}\text { Risk due to inadequate knowledge of } \\
\text { cloud }\end{array}$ \\
\hline Shared technology & Attacks due to shared resources. \\
\hline Shared technology & Attacks due to shared resources. \\
\hline
\end{tabular}

\section{CONCLUSION}

In this paper, we audit the situation of the present and expected destiny bearings for the coordination of a long way flung gazing of healing innovation into clinical act of drugs. Wearable sensors, specifically the ones furnished with IOT perception, gives fascinating alternatives to empower the perception and recording of records at domestic and workplaces, over a greater drawn out duration than is presently achieved inside the workplace and studies facility visits. It's a fortune trove of records, while investigated and displayed to the health practitioner in imagining efficaciously take in can probably profoundly enhance wellness and decrease charges. We likewise have referenced severa safety demanding situations IOT. All systems offer and assure the security of statistics on every personal and open mists. A bit picture of the IOT is now apparent inside the gift unfold wherein the detecting system being recognized with the net, and IP-primarily based improvements critical to provide ordinary and wellacknowledged reason for developing and conveying new IOT packages. Understand that the critical prerequisite is to create units for secure correspondence and for some, cloudprimarily based totally packages

\section{REFERENCES}

1. X. L. D. A. Li, Shancang and S. Zhao, "The net of things: an define," Frontiers of records structures, vol. 17, no. 2, pp. 243-259, April 2015.

2. W. Stallings, current network basis: SDN, NFV, QoE, IOT, and Cloud. Addison-Wesley professional, 2015.

3. Gubbi, R. Buyya, S. Marusic, and M. Palaniswami, "net of factors (bonehead): A dream, engineering components, and future headings," FutureGeneration pc structures, vol. 29, no. 7, pp 1645 - . 1660, 2013A. Abuarqoub, M. Hammoudeh, B. Adebisi, S. Jabbar, A. Bounceur, and Al-Assad, "grouping and dynamic a long way flung portable management

4. Holler, V. Tsiatsis, C. Mulligan, S. Karnouskos, S. Avesand, and D. Boyle, From the machine-to-device for the internet of factors: an acquaintance tion with the brand new Age of Intelligence. Amsterdam: Elsevier, 2014.

5. Tan and N. Wang, " destiny net: internet of factors, " in Proc.Third Int. Conf. Adv. Comput. Hypothesis Eng. (ICACTE), vol. August 5th, 2010, p. V5-376 V5-380.

6. Z. Pain, " technology and layout of the internet-of-things (IOT) for the wellness and prosperity, " M.S. Notion, Dept. Of Electron. Comput. Syst., KTH-Roy. Inst. Technol., Stockholm, Sweden, January 2013

7. W. Zhao, C. Wang, and Y. Nakahira, "healing packages inside the internet of things," at the IET Int. Conf. On Com. Tech. Furthermore, programs (ICCTA 2011), October 2011, p. 660-665.

8. Hu, D. Xie, and S. Shen, "On the usage of net of things inside the subject of medicinal and human services," in IEEE Int. Conf. Additionally, IEEE Cyber, physical and Social Computing green Computing and Communications (GreenCom), (iThings/CPSCom), August 2013, p. 2053-2058.

9. T. Soyata, R. Muraleedharan, C. Funai, M. Kwon, and W. Heinzelman, "Cloud-imaginative and prescient: realTime Face acknowledgment using quickening engineering cellular little tremendous Cloud," in complaints of the 17th IEEE Symposium on computer systems and Communications (ISCC IEEE 2012), Cappadocia, Turkey, Jul 2012, p. 59-sixty six.

10. Nalinipriya and Aswin Kumar R., "vast therapeutic information stockpiling with a symmetric calculation that stands apart over the mists - ensured structure," in IEEE Int. Conf. On clever structures and structures (ICSSS), March 2013, p. 171-177.

11. F. M. Hani, I. V. Paputungan, M. F. Hassan, V. S. Asirvadam, and Daharus, "Self-focus Disbursed garage for healing photo test out statistics, "in Int.Conf. On laptop and Inf. Technological know-how (ICCOINS), June 2014, p. 1-6. 